\title{
Nonnegative Integral Subset Representations of Integer Sets
}

\author{
Michael J. Collins * $\quad$ David Kempe ${ }^{\dagger} \quad$ Jared Saia $^{\ddagger} \quad$ Maxwell Young ${ }^{\ddagger}$
}

\begin{abstract}
We consider an integer-subset representation problem motivated by a medical application in radiation therapy. We prove NP-completeness, derive nontrivial bounds, and report on the performance of a greedy heuristic.

Keywords: approximation algorithms; combinatorial problems; heuristics; additive number theory; radiology
\end{abstract}

\section{Introduction}

Given a set of positive integers $S=\left\{s_{1}<s_{2}<\cdots<s_{n}\right\}$, the Generating SET problem consists of finding a minimum size set of positive integers $T=\left\{t_{1}<\right.$ $\left.t_{2}<\cdots<t_{m}\right\}$ such that every element of $S$ is the sum of a subset of $T$. Such a set $T$ is called a "generating set" for $S$; note that $T$ does not have to be a subset of $S$. In addition to being a very natural problem in combinatorial number theory, this problem is motivated by medical applications in planning radiation therapy: elements of $S$ represent radiation dosages required at various points, while an element of $T$ represents a dose delivered simultaneously to multiple points. We seek to minimize the number of steps required to deliver exactly the specified dose to each point. There is no need to allow $T$ to be a multiset; if some $t$ appears twice in $T$, we can w.l.o.g. replace the second appearance with $2 t$. Somewhat similar optimization problems arising from radiation therapy are considered in $[1,3,4]$. The case in which the elements of $T$ can be negative or fractional is considered in $[2,5,6]$. Trivially $\lceil\log n\rceil \leq m \leq \min \left(n,\left\lceil\log s_{n}\right\rceil\right)$, since $T$ must be large enough to have $n$ distinct subsets, and $S$ can be represented by itself or by $\left\{1,2,4, \ldots 2^{\left\lfloor\log s_{n}\right\rfloor}\right\}$.

\footnotetext{
*Sandia National Laboratories, Albuquerque, NM, USA; email: mjcolli@sandia.gov

${ }^{\dagger}$ Dept. of Computer Science, University of Southern California, CA, USA; email: ckempe@ usc.edu

${ }^{\ddagger}$ Dept. of Computer Science, University of New Mexico, NM, USA; email: saia@cs.unm.edu, young@cs.unm.edu
} 


\section{NP-Completeness}

In this section, we prove the following decision version of the Generating SeT problem to be NP-complete.

Definition 1. Generating Set:

Input: A target set $S$ of positive integers, and a non-negative integer $k$.

Question: Is there a set $T$ of positive integers such that $|T|=k$, and every element $s \in S$ is the sum of some subset of the elements in $T$ ?

We prove that Generating Set is NP-complete via a reduction from Positive NAE-3SAT(5), a variant of NAE-3SAT, which is proven NP-complete in [7]. Reduction from SUBSET SUM might appear to be more natural, but no such reduction is known.

Definition 2. Positive NAE-3SAT(5):

Input: $A$ SAT formula $\phi$ with $n \geq 5$ variables and $m$ clauses. Each clause contains exactly 3 distinct and uncomplemented variables.

Question: Is there a truth assignment such that each clause of $\phi$ contains both a true variable and a false variable?

Theorem 1. Generating Set is NP-complete.

Our reduction takes an instance $\phi$ of Positive NAE-3SAT(5), with variables labeled $x_{1}, \ldots, x_{n}$ and $m$ clauses, and constructs an instance of Generating Set in the following way:

- Add 1 to $S$.

- For each variable $x_{i}$, add $a_{i}:=n^{i}$ to $S$. We call $a_{i}$ the "variable number" for $x_{i}$.

- For each clause $c$ containing the variables $x_{i}, x_{j}, x_{k}$, add the $y_{c}:=a_{i}+a_{j}+$ $a_{k}-1=n^{i}+n^{j}+n^{k}-1$ to $S$. We call $y_{c}$ the "clause number" for $c$.

The decision question of Generating Set is then whether the set $S$ can be represented by an integer set of size at most $n+1$.

We begin by establishing useful lemmas about the possible structures of generating sets $T$. In the sequel, we assume that $\phi$ is fixed (and has $n \geq 5$ variables), and we always use $S$ to refer to the set generated by the reduction. We also always assume that $T=\left\{1 \leq b_{1} \leq b_{2} \leq \ldots \leq b_{n}\right\}$ is a generating set for $S$ of size $n+1$.

Lemma 1. For all $i \leq n$, we have $a_{i}-\sum_{j=1}^{i-1} a_{j}-1 \leq b_{i} \leq a_{i}$.

Proof. First notice that 1 must be in $T$. Second, assume that $b_{i}>a_{i}$ for some $i$, and let $r$ be the smallest such index. Because $b_{i} \leq a_{i}$ for $i \leq r-1$, we have 
$1+\sum_{i=1}^{r-1} b_{i} \leq 1+\sum_{i=1}^{r-1} a_{i}<n^{r}=a_{r}$ (for $n \geq 2$ ). This implies that $T$ cannot generate $a_{r}$, which is a contradiction. Thus, $b_{i} \leq a_{i}$ for all $i$.

Next, assume that $b_{i}<a_{i}-\sum_{j=1}^{i-1} a_{j}-1$ for some $i$, and let $r$ be the largest index such that $b_{r}<a_{r}-\sum_{i=1}^{r-1} a_{i}-1$. Then, $b_{r+1} \geq a_{r+1}-\sum_{i=1}^{r} a_{i}-1>a_{r}$ (for $n \geq 3)$. This implies that $a_{r}$ is the sum of elements of some subset of $\left\{1, b_{1}, \ldots, b_{r}\right\}$. But $1+\sum_{i=1}^{r} b_{i} \leq a_{r}-1$, which means that $T$ cannot generate $a_{r}$, which is a contradiction. Thus, for all $i, b_{i} \geq a_{i}-\sum_{j=1}^{i-1} a_{j}-1$.

Lemma 2. Let $\mathbf{b}=\left(b_{1}, b_{2}, \ldots, b_{n}\right)$, and $X=\{0,1,2,3\}^{n}$. Then, for any two distinct vectors $\mathbf{u}, \mathbf{v} \in X,|\mathbf{u} \cdot \mathbf{b}-\mathbf{v} \cdot \mathbf{b}| \geq 3$.

Proof. Let $\mathbf{u}=\left(u_{1}, u_{2}, \ldots, u_{n}\right)$ and $\mathbf{v}=\left(v_{1}, v_{2}, \ldots, v_{n}\right)$, and $k$ be the largest index such that $u_{k} \neq v_{k}$. Without loss of generality, $u_{k}>v_{k}$. Then, using Lemma 1 , $|\mathbf{u} \cdot \mathbf{b}-\mathbf{v} \cdot \mathbf{b}| \geq \mathbf{u} \cdot \mathbf{b}-\mathbf{v} \cdot \mathbf{b} \geq b_{k}-3 \sum_{i<k} b_{i} \geq\left(a_{k}-\sum_{i<k} a_{i}-1\right)-3 \sum_{i<k} a_{i}=$ $n^{k}-\frac{4 n^{k}-3 n-1}{n-1} \geq 3$, for $n \geq 5$.

Corollary 1. For all $i \leq n, T$ generates either $a_{i}$ or $a_{i}-1$, but not both, without using 1 .

Proof. Apply Lemma 2, taking $\mathbf{u}, \mathbf{v}$ to be the $0-1$ vectors describing which elements of $T$ are used to generate $a_{i}$ and $a_{i}-1$, and notice that $\left|a_{i}-\left(a_{i}-1\right)\right|=1$.

Lemma 3. For any "clause number" $y_{c}=a_{i}+a_{j}+a_{k}-1$ in $S$, the following two statements are true:

1. At least one of $\left\{a_{i}, a_{j}, a_{k}\right\}$ can be generated by $T$ without using 1 .

2. There is an $a \in\left\{a_{i}, a_{j}, a_{k}\right\}$ such that $T$ generates $a-1$ without using 1 .

Proof. If each of $a_{i}, a_{j}, a_{k}$ requires 1 to be generated, then taking the corresponding incidence vectors $\mathbf{v}_{\mathbf{i}}, \mathbf{v}_{\mathbf{j}}, \mathbf{v}_{\mathbf{k}}$ for the elements of $T$ except 1 , and writing $\mathbf{v}:=\mathbf{v}_{\mathbf{i}}+\mathbf{v}_{\mathbf{j}}+\mathbf{v}_{\mathbf{k}}$, we find that $\mathbf{v} \cdot \mathbf{b}=a_{i}+a_{j}+a_{k}-3$. Now, by Lemma $2, T$ cannot generate $a_{i}+a_{j}+a_{k}-2$ or $a_{i}+a_{j}+a_{k}-1$ without the number 1 , and hence cannot generate $a_{i}+a_{j}+a_{k}-1$ at all, a contradiction. Similarly, if all of $a_{i}, a_{j}, a_{k}$ can be generated without using the number 1 , the analogous vector $\mathbf{u}$ satisfies $\mathbf{u} \cdot \mathbf{b}=a_{i}+a_{j}+a_{k}$. Again, by Lemma $2, a_{i}+a_{j}+a_{k}-1$ is not generated by $T$, a contradiction.

We are now ready to prove Theorem 1 :

Proof of Theorem 1. It is clear that Generating Set is in NP, and the reduction clearly runs in polynomial time. To prove the correctness of the reduction, first assume that we have an assignment such that each clause of $\phi$ has either one or 
two true variables. We create $T$ in the following way. First, $T$ always contains the number 1 . In addition, for each $i$, if $x_{i}=$ true in the satisfying truth assignment, $T$ contains the number $n^{i}$; otherwise, it contains the number $n^{i}-1$. Clearly, $T$ generates all "variable numbers" $a_{i}$, either simply as $a_{i}=n^{i}$, or as $a_{i}=\left(n^{i}-1\right)+1$. Since every clause $c$ has either one or two true variables, summing the values corresponding to the variables will give either $a_{i}+a_{j}+a_{k}-1$ or $a_{i}+a_{j}+a_{k}-2$. In the former case, $y_{c}$ is already generated, and in the latter case, it is generated by adding 1 .

Conversely, if $T$ is a generating set of size $n+1$, we define a truth assignment to the $x_{i}$ by setting $x_{i}=$ true if $T$ generates $a_{i}$ without using the number 1 , and $x_{i}=$ false otherwise (in which case $T$ generates $a_{i}$ using the number 1 , by Corollary 1). By Lemma 3, for each clause $c$, the fact that $T$ can generate the value $y_{c}=a_{i}+a_{j}+a_{k}-1$ guarantees that the truth assignment received by the corresponding clause contains at least one variable set to true, and at least one variable set to false, completing the proof.

\section{Lower Bounds on $m$}

The trivial lower bound from the introduction can be improved in some cases. Let $|T|=m$, and define the multiset of differences

$$
D_{S}=\left\{s_{j}-s_{i}: i<j \leq n\right\} .
$$

Let $d_{S}$ be the number of distinct elements in $D_{S}$. At most $\frac{3^{m}-1}{2}$ positive differences can be generated as the difference between two subsets of $T$; in such a difference, each $t_{i}$ appears positively, negatively, or not at all, and half of the nonzero differences are negative. Thus

$$
m \geq \log _{3} 2 d_{S}>0.63 \log 2 d_{S}
$$

(where all logs are base 2 unless indicated otherwise). In particular, if $d_{S}=\alpha n^{2} / 2$, we have

$$
m>1.26 \log n-0.63 \log \frac{1}{\alpha} .
$$

More generally, there are fewer than $(k+1)^{m}$ distinct sums of $k$ subsets of $T$, so this must be larger than the number of distinct values of sums of $k$ elements of $S$ (allowing multisets). Then, we have

Theorem 2. If there are $\alpha \frac{n^{k}}{k !}$ distinct sums of $k$ elements of $S$ and $T$ generates $S$, then

$$
m>\frac{k}{\log (k+1)} \log n-\frac{\log k !}{\log (k+1)}-\frac{\log \alpha^{-1}}{\log (k+1)} .
$$

We can also obtain lower bounds of $1+\lceil\log n\rceil$ by considering parities. Let $n^{\prime}$ be the number of odd values in $S$; if $n^{\prime} \neq 0$ then there must be at least one odd 
element in $T$, so exactly $2^{m-1}$ subsets of $T$ have odd sums. Thus, $2^{m-1}$ must be at least $\max \left(n^{\prime}, n-n^{\prime}\right)$, and

$$
m \geq 1+\left\lceil\log \max \left(n^{\prime}, n-n^{\prime}\right)\right\rceil .
$$

More generally, consider the values in $S \bmod M$. If any $s_{i}$ is not zero $\bmod M$, then some $t_{j}$ is nonzero $\bmod M$, thus no more than half of the subsets of $T$ can have the same value $\bmod M$ (since adding or removing $t_{j}$ changes the value of a set $\bmod M$ ), and $m \geq 1+\lceil\log \hat{n}\rceil$ where $\hat{n}$ is the size of the largest equivalence class $\bmod M$.

\section{Fraction of Instances Requiring Large Representa- tions}

If we are interested in instances whose numbers are bounded by $2^{k}$, and $n$ is sufficiently large relative to $k$, then most of these instances will not have small generating sets (i.e., sublinear in $k$ ). Specifically, there are at most $\left(\begin{array}{c}2^{k} \\ j\end{array}\right)$ sets $T$ of size $j$, each generating at most $2^{j}$ distinct sums. The only $n$-element sets generated by $T$ are its $n$-element subsets. Therefore, there are at most

$$
\left(\begin{array}{c}
2^{k} \\
j
\end{array}\right) \cdot\left(\begin{array}{c}
2^{j} \\
n
\end{array}\right)
$$

subsets of size $n$ that can be generated by sets $T$ of size at most $j$. On the other hand, the total number of problem instances of size $n$ is $\left(\begin{array}{c}2^{k} \\ n\end{array}\right)$, and using the bounds $\left(\frac{a}{b}\right)^{b} \leq\left(\begin{array}{l}a \\ b\end{array}\right) \leq\left(\frac{e a}{b}\right)^{b}$, the fraction of instances that can be generated by sets of size at most $j$ is at most

$$
2^{j k+j n+(\log e)(j+n)-k n-j \log j} .
$$

By taking limits for $k, n$, we obtain the following:

Theorem 3. Fix $\alpha<1$, and consider problem instances of $n$ numbers bounded by $2^{k}$.

1. If $\frac{n}{k} \rightarrow \infty$ as $k, n \rightarrow \infty$, the fraction of problem instances representable with $|T| \leq \alpha k$ converges to zero.

2. If $\frac{n}{k} \rightarrow 0$ as $k, n \rightarrow \infty$, the fraction of problem instances representable with $|T| \leq \alpha$ converges to zero.

For example, if we randomly select fifty sixteen-bit integers (so that trivially $6 \leq|T| \leq 16$ ), the probability of being able to represent this set with $|T| \leq 11$ is less than one in seventeen million. 


\section{A Greedy Heuristic}

We propose a greedy heuristic for constructing a representing set $T$. The idea is that at each step, we choose a $t$ which will enable us to represent the largest number of $s \in S$ which have not been represented already.

Start with $T=\emptyset$. In the first iteration, find the most common difference $d$ in $D_{S}$ and set $T_{1}=\{d\}$. For all $s_{j}$ such that $s_{j}-s_{i}=d$ for some $i$, remove $s_{j}$ from $S$. The idea is that after we have removed $s_{j}$, the representation of $s_{j}$ will be $d$ plus the representation we eventually find for $s_{i}$. We say that $s_{j}$ has been "retracted" into $s_{i}$. Let $R_{i}$ be the representation of $s_{i}$, i.e. the subset of $T$ such that $s_{i}=\sum_{t \in R_{i}} t$. So at this point we have the partial representation $R_{j}=\{d\} \cup R_{i}$. For notational convenience we let $s_{1}=0, R_{1}=\emptyset$. When $s_{j}$ is retracted into zero, or into an $s_{i}$ whose representation was completed in a previous iteration, its representation is complete (as is the representation of anything that depends, directly or indirectly, upon $R_{j}$ ).

One additional detail is that we might not be able to simultaneously remove all such $s_{j}$, we can only remove $s_{j}$ if we do not remove $s_{i}$ (otherwise we would need two copies of $d$ in $T$ ). This situation arises if $d$ appears twice consecutively as a difference, $s_{j}=s_{i}+d$ and $s_{k}=s_{j}+d$. In such a sequence of repeated adjacent differences we can only take alternating elements out of $S$. We take this into account in selecting the best $d$.

In subsequent iterations we do not only look at differences among the remaining elements of $S$, but we also look at how the elements already in $T$ can be used to build representations. We can have

$$
s_{j}=s_{i}-t+t^{\prime}+d
$$

for any $t \in R_{i}$ and any $t^{\prime} \in T-R_{i}$ such that

- there is no $s_{k}$ whose current representation includes both $t^{\prime}$ and $R_{j}$ (otherwise $t^{\prime}$ would occur twice in $R_{k}$ )

- there is no $s_{k}$ whose current representation uses $R_{j}$ and intersects $R_{i}-\{t\}$ (otherwise elements of $R_{k} \cap\left(R_{i}-\{t\}\right)$ would occur twice in $R_{k}$ )

With proper bookkeeping we can enumerate all allowable choices of $\left(i, j, t, t^{\prime}\right)$ for which $d>0$ in (3). We also do not consider retraction into any $s_{i}$ that has a partial representation: $s_{i}$ must either have a completed representation, or not yet have any representation. Retraction into partially-represented elements would require additional bookkeeping and more complex restrictions on $t$ and $t^{\prime}$. Having enumerated all possibilities we add $d^{*}$ to $T$, where $d^{*}$ is the value which enables us to retract the largest number of $s_{j}$.

Of course we are not limited to a single $t$ or $t^{\prime}$, we could consider arbitrary subsets of $R_{i}$ and of $T-R_{i}$. But this leads to exponential growth in the number 


\begin{tabular}{|c|c|c|c|}
\hline $\mathrm{n}$ & $\left\lceil\log s_{n}\right\rceil$ & Avg. Heuristic & pct. below trivial \\
\hline 8 & 6 & 5.42 & 57 \\
10 & 6 & 5.76 & 24 \\
15 & 6 & 6.0 & 0 \\
10 & 8 & 6.98 & 90 \\
15 & 8 & 7.83 & 16 \\
20 & 8 & 7.98 & 2 \\
15 & 12 & 10.42 & 93 \\
20 & 12 & 11.38 & 59 \\
30 & 12 & 12.0 & 0 \\
25 & 16 & 15.28 & 64 \\
30 & 16 & 15.91 & 9 \\
40 & 16 & 16.0 & 0 \\
\hline
\end{tabular}

Table 1: Results for instances uniformly randomly generated between 1 and $2^{m}-1$; 100 trials each.

\begin{tabular}{|c|c|c|c|}
\hline $\mathrm{n}$ & $\left\lceil\log s_{n}\right\rceil$ & Avg. Heuristic & pct. below trivial \\
\hline 15 & 12 & 9.52 & 100 \\
20 & 12 & 10.09 & 99 \\
30 & 12 & 11.06 & 69 \\
25 & 16 & 14.85 & 79 \\
30 & 16 & 15.69 & 28 \\
40 & 16 & 15.99 & 1 \\
\hline
\end{tabular}

Table 2: Results for instances generated by random subsets of $\{1,2,8,32,64,256$, $1024,2048\}$ (12-bit $\left.s_{n}\right)$ and $\{1,4,8,16,64,128,512,1024,2048,8192,16384,32768\}$ (16-bit $\left.s_{n}\right) ; 100$ trials each.

of options to consider. To maintain a polynomial running time we must limit the size of the subsets by a constant; the implementation used for the results of the next section considers pairs of subsets with union of size at most 3 . Thus we may consider representations such as $s_{j}=s_{i}-t_{1}-t_{2}-t_{3}+d$ or $s_{j}=s_{i}-t+t_{1}^{\prime}+t_{2}^{\prime}+d$ where $t_{i} \in R_{i}$ and $t_{i}^{\prime} \in T-R_{i}$ satisfy the conditions above.

\subsection{Experimental Results}

We uniformly randomly generated sets of $n m$-bit numbers for $n, m$ as shown in Table 1, computing the average size (over 100 random instances) of the representation found by the heuristic and the fraction of instances for which a better-than-trivial solution was obtained. 
These results in Table 1 are difficult to interpret since we do not know what fraction of instances should have better-than-trivial solutions. We generated instances known to have nontrivial solutions by taking sums of random subsets of a set of generators of size less than $\log s_{n}$. In this case it is clear that the heuristic is rarely finding an optimal solution (which would be no larger than the known generating set), but it tends to find smaller generating sets than were found for uniformly generated instances. In no case did we find a generating set smaller than the set actually used to create the instance.

\section{Acknowledgments}

We would like to thank Shuang Luan, Cris Moore, and MohammadReza Salavatipour for useful discussions.

\section{References}

[1] N. Bansal, D. Coppersmith, and B. Schieber. Minimizing setup and beam-on times in radiation therapy. In Proceedings of APPROX 2006, 2006.

[2] M. Develin. Optimal subset representations of integer sets. Journal of Number Theory, 89:212-221, 2001.

[3] T. Kalinowski. The algorithmic complexity of the minimization of the number of segments in multileaf collimator field segmentation. preprint.

[4] S. Luan, D.Z. Chen, X.S. Hu, S.A. Naqvi, C. Wang, and C.X. Yu. Generalized geometric approaches for leaf sequencing problems in radiation therapy. International Journal of Computational Geometry and Applications (IJCGA), 16:175-204, 2006.

[5] D. Mills. Some observations on subset sum representations. preprint.

[6] D. Moulton and D. Petrie. Representing powers of numbers as subset sums of small sets. Journal of Number Theory, 89:193-211, 2001.

[7] T. Schaefer. The complexity of satisfiability problems. In Proc. 10th ACM Symp. on Theory of Computing, pages 216-226, 1978. 